\title{
Congruence structure of planar semimodular lattices: The General Swing Lemma
}

\author{
GÁbor Czédli, George Grätzer, AND Harry Lakser \\ To the memory of E. T. Schmidt
}

\begin{abstract}
The Swing Lemma (G. Grätzer, 2015) describes how a congruence spreads from a prime interval to another in a slim (having no $M_{3}$ sublattice), planar, semimodular lattice. We generalize the Swing Lemma to planar semimodular lattices.
\end{abstract}

\section{Introduction}

Congruences in a distributive lattice $L$ were investigated in G. Grätzer and E. T. Schmidt [29]: for $a \leq b, c \leq d \in L$, the interval $[a, b]$ congruence spreads to the interval $[c, d]$ (that is, $c \equiv d(\bmod \operatorname{con}(a, b))$ or equivalently, $\operatorname{con}(a, b) \geq$ $\operatorname{con}(c, d))$ iff we can get from $[a, b]$ to $[c, d]$ with one up-step (joining with an element) and one down-step (meeting with an element). See G. Grätzer [10] for an interesting application of this idea.

In G. Grätzer and E. T. Schmidt [30] and G. Grätzer, H. Lakser, and E. T. Schmidt [28], we succeeded in representing a finite distributive lattice $D$ as the congruence lattice of a finite semimodular lattice $L$. It was a great surprise that the lattice $L$ constructed was planar.

This result started the study of planar semimodular lattices in G. Grätzer and E. Knapp [26], [27]; see also G. Czédli and E. T. Schmidt [7], [8], G. Czédli [1]. For a 2013 review of this field, see G. Czédli and G. Grätzer [5]. Part VII in the book G. Grätzer [16] provides a 2015 review of this field. More articles on these topics are listed in the Bibliography.

It is a special focus of this new field to examine how a prime interval $\mathfrak{p}$ congruence spreads to another prime interval $\mathfrak{q}$.

The first main result of this type, the Trajectory Coloring Theorem for Slim Rectangular Lattices, is due to G. Czédli [3]. It gives a quasiordering of trajectories of slim rectangular lattices (a subclass of slim, planar, semimodular lattices) $L$ to represent the ordered set of join-irreducible congruences of $L$.

The second main result of this type is the Swing Lemma for slim (having no $\mathrm{M}_{3}$ sublattice), planar, semimodular lattices in G. Grätzer [22], see Section 3.

In this paper, we extend the Swing Lemma to planar semimodular lattices. We use two different approaches.

2010 Mathematics Subject Classification: Primary: 06C10, Secondary: 06B05.

Key words and phrases: Lattice, congruence, semimodular slim, planar, Swing Lemma. This research was supported by NFSR of Hungary (OTKA), grant number K 115518. 
The first, utilizes the first author's Eye Lemma [1, Lemma 5.2], a contribution to the theory of congruences of planar lattices. This lemma is applied to planar semimodular lattices in Lemma 11.

The second approach is a contribution to the theory of congruences of finite lattices. Instead of the Eye Lemma, we prove the Tab Lemma for finite lattices, which is based on the Prime-Projectivity Lemma of the second author [12, Lemma 4]. We state and prove Lemma 8, a general version of the Swing Lemma that does not require planarity.

1.1. Outline. The basic concepts and results are introduced in Section 2.

In Section 3, we state the Swing Lemma and the General Swing Lemma. Section 4 presents our short approach through planar lattices, utilizing the Eye Lemma. The Tab Lemma for finite lattices is stated and proved for general finite lattices in Section 5, which is then applied in Section 6 to prove the General Swing Lemma. Finally, Section 7 discusses some related topic, including a formulation of the General Swing Lemma that makes no reference to planarity.

\section{Preliminaries}

We use the basic concepts and notation as in the books G. Grätzer [11] and $[16]$.

We call a planar semimodular lattice a PS lattice and a slim, planar, semimodular lattice an SPS lattice. Note that a planar lattice is finite by definition.

For a lattice $L$, let Prime $(L)$ denote the set of prime intervals of $L$.

A multi-diamond is a lattice $M$ isomorphic to the $(n+2)$-element modular lattice $\mathrm{M}_{n}$ of length 2 for some integer $n \geq 3$. A covering multi-diamond $M$ in a lattice $L$ is an interval in $L$ isomorphic to some lattice $\mathrm{M}_{n}$ for some integer $n \geq 3$. An element $m \in L$ is a $t a b$ of $L$ if it is doubly-irreducible and it is in a covering multi-diamond in $L$. Note that $m^{*}$, the unique upper cover of $m$, is the unit of the multi-diamond and $m_{*}$, the unique lower cover of $m$, is the zero.

Definition 1. Let $\mathfrak{p}, \mathfrak{q}$ be prime intervals in a lattice $L$. We say that $\mathfrak{p}$ swings to $\mathfrak{q}$ if the following conditions are satisfied:

(i) $\mathfrak{p} \neq \mathfrak{q}$;

(ii) $1_{\mathfrak{p}}=1_{\mathfrak{q}}$;

(iii) there is a lower cover $a \neq 0_{\mathfrak{p}}, 0_{\mathfrak{q}}$ of $1_{\mathfrak{p}}$ such that the three element set $\left\{0_{\mathfrak{p}}, 0_{\mathfrak{p}}, a\right\}$ generates a sublattice $S$ of $L$ isomorphic to $\mathrm{S}_{7}$, where $0_{\mathfrak{q}}$ is the unique dual atom of $S$ that is a proper join-see Figure 1.

This swing concept-modified from G. Grätzer [22], where it is defined for SPS lattices - makes sense in an arbitrary lattice, and we get the following statement for general lattices, equally as trivial as it is for SPS lattices.

Lemma 1. Let $L$ be any lattice, let $\mathfrak{p}, \mathfrak{q}$ be prime intervals in $L$, and let $\mathfrak{p}$ swing to $\mathfrak{q}$. Then $\mathfrak{p}$ congruence spreads to $\mathfrak{q}$. 


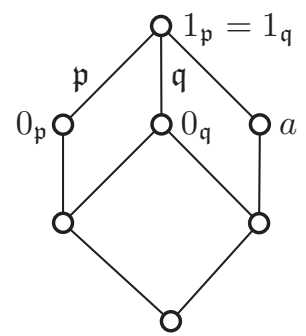

FIGURE $1 . \mathfrak{p}$ swings to $\mathfrak{q}$

We shall need the following result later on.

Lemma 2. Let $L$ be a finite semimodular lattice, let $[o, i]$ be an interval of length 2 in $L$, and let $m$ be an atom of $[o, i]$ that is join-irreducible in $L$. Let $x \notin[o, i]$ be a lower cover of $i$ in $L$. Then there is an atom $a \in[o, i]$ such that the elements $x, a, m$ generate a sublattice $S$ of $L$ that is isomorphic to $S_{7}$, where $a$ is the dual atom of $S$ that is a proper join.

Proof. Since $x \nsupseteq m, u=x \wedge m<m$. Since $m$ is join-irreducible in $L$, it has exactly one lower cover $o$, hence, $u \leq o$. Since $x \nsupseteq o$, we conclude that $u<o$.

Since $x$ is a lower cover of $i$, it must be distinct from $u$ and so $u<x$. Then there is an upper cover $v \leq x$ of $u$. Since $x \wedge o=u$, it follows that $v \not \leq o$ - see Figure 2. Thus, by semimodularity, $a=v \vee o \leq i$ covers $o$. Since $a$ is proper join, it is distinct from $m$. Note that $a \in[o, i]$, so it is distinct from $x$. Since the length of $[o, i]$ is 2 , it follows that $a$ is a lower cover of $i$. Set $y=x \wedge a \geq v$.

Then the subset $S=\{u, y, o, x, a, m, i\}$ is the sublattice generated by the set of lower covers $\{x, a, m\}$ of $i$, and is isomorphic to $S_{7}$ with the dual atom $a$ a proper join.

Indeed, as noted above, the lower covers $x, a, m$ of $i$ are all distinct.

Furthermore, $S$ is a meet subsemilattice of $L$. By definition, $x \wedge a=y$; also $a \wedge m=o$, since they are distinct upper covers of $o$, and $x \wedge m=u$, again by definition.

That $S$ is also a join subsemilattice of $L$ is also easy:

$$
x \vee a=a \vee m=x \vee m=i
$$

since $x, a, m$ are distinct lower covers of $i$. Note also that by definition, $a=v \vee o$ and also

$$
a=v \vee o \leq y \vee o \leq a,
$$

that is, that $y \vee o=a$.

The other two incomparable joins are then immediate:

$$
y \vee m=y \vee o \vee m=a \vee m=i,
$$

and, similarly,

$$
o \vee x=o \vee y \vee x=a \vee x=i .
$$


See Figure 2, and note that, although $v$ appears in the figure, it is not an element of $S$.

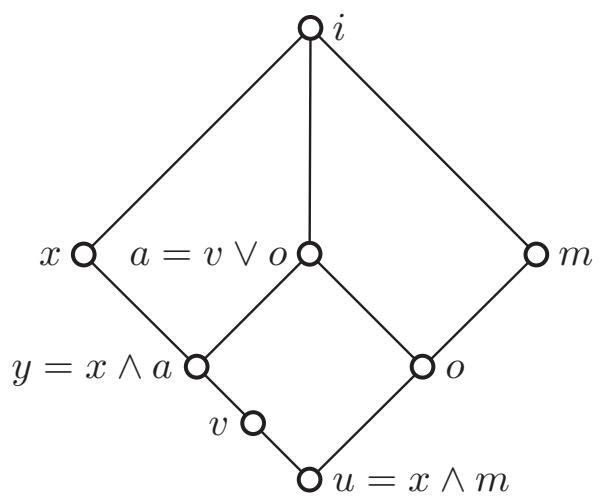

Figure 2. Lemma 2: Generating the $S_{7}$.

For prime intervals $\mathfrak{p}, \mathfrak{q}$ in an SPS lattice $L$, if $\mathfrak{p}$ congruence spreads to $\mathfrak{q}$, then the Swing Lemma gives a special kind of projectivity. We call such a projectivity an SPS projectivity. Since the concept "p swings to q" now makes sense in any lattice, we can define the concept of SPS projectivity for any lattice.

Definition 2. Let $L$ be a lattice, and let $\mathfrak{p}, \mathfrak{q}$ be prime intervals in $L$. We say that $\mathfrak{p}$ is SPS projective to $\mathfrak{q}$ if either $\mathfrak{p}=\mathfrak{q}$ or there exists a prime interval $\mathfrak{r}$ with $\mathfrak{p}$ up-perspective to $\mathfrak{r}$, and a sequence of prime intervals and a sequence of binary relations

$$
\mathfrak{r}=\mathfrak{r}_{0} \varrho_{1} \mathfrak{r}_{1} \varrho_{2} \mathfrak{r}_{2} \cdots \mathfrak{r}_{n-1} \varrho_{n} \mathfrak{r}_{n}=\mathfrak{q}
$$

where each relation $\varrho_{k}$ is either a down perspectivity of $\mathfrak{r}_{k}$ to $\mathfrak{r}_{k+1}$ or a swing of $\mathfrak{r}_{k}$ to $\mathfrak{r}_{k+1}$.

We call (1) an SPS sequence. By Lemma 1, the following holds in any lattice.

Lemma 3. Let $L$ be any lattice, and let $\mathfrak{p}$ and $\mathfrak{q}$ be prime intervals in $L$. If $\mathfrak{p}$ is SPS projective to $\mathfrak{q}$, then $\mathfrak{p}$ congruence spreads to $\mathfrak{q}$.

We will need the following results (G. Grätzer [21] and [16, Lemma 3.3]):

Lemma 4. Let $L$ be a finite lattice. Let $\boldsymbol{\gamma}$ be an equivalence relation on $L$ all of whose equivalence classes are intervals. Then $\gamma$ is a congruence relation iff the following condition and its dual hold:

If $x$ is covered by $y \neq z \in L$ and $x \equiv y(\bmod \gamma)$, then $z \equiv y \vee z(\bmod \gamma)$.

This lemma is easy to prove, but it is useful. The next lemma is far deeper (G. Grätzer [12] and [16, Lemma 24.1]). To state it, we need the concept of 
prime-perspectivity. Recall that for (not necessarily prime) intervals $I=\left[0_{I}, 1_{I}\right]$ and $J=\left[0_{J}, 1_{J}\right]$ we say that $I$ is down perspective to $J$, in formula $I \stackrel{\text { dn }}{\sim} J$, if $0_{I} \vee 1_{J}=1_{I}$ and $0_{I} \wedge 1_{J}=0_{J}$.

Let $L$ be a finite lattice and let $\mathfrak{p}$ and $\mathfrak{q}$ be prime intervals of $L$. Then the binary relation $\mathfrak{p}$ prime-perspective down to $\mathfrak{q}$, in formula, $\mathfrak{p} \stackrel{\mathrm{p}-\mathrm{dn}}{\longrightarrow} \mathfrak{q}$, is defined as $\mathfrak{p} \stackrel{\text { dn }}{\sim}\left[0_{\mathfrak{p}} \wedge 1_{\mathfrak{q}}, 1_{\mathfrak{q}}\right]$ (that is, $0_{\mathfrak{p}} \vee 1_{\mathfrak{q}}=1_{\mathfrak{p}}$ ) and $0_{\mathfrak{p}} \wedge 1_{\mathfrak{q}} \leq 0_{\mathfrak{q}}$; we define prime-perspective up, $\mathfrak{p} \stackrel{\mathrm{p} \text {-up }}{\longrightarrow} \mathfrak{q}$, dually - see Figure 3. Let prime-perspective, $\mathfrak{p} \stackrel{\mathrm{p}}{\longrightarrow} \mathfrak{q}$, mean that $\mathfrak{p} \stackrel{\mathrm{p} \text {-up }}{\longrightarrow} \mathfrak{q}$ or $\mathfrak{p} \stackrel{\mathrm{p} \text {-dn }}{\longrightarrow} \mathfrak{q}$ and let prime-projective, $\mathfrak{p} \stackrel{\mathrm{p}}{\Longrightarrow} \mathfrak{q}$, be the transitive extension of $\stackrel{\mathrm{p}}{\longrightarrow}$.

Lemma 5 (Prime-Projectivity Lemma). Let $L$ be a finite lattice and let $\mathfrak{p}$ and $\mathfrak{q}$ be distinct prime intervals in $L$. Then $\mathfrak{q}$ is collapsed by $\operatorname{con}(\mathfrak{p})$ iff $\mathfrak{p} \stackrel{p}{\Longrightarrow} \mathfrak{q}$, that is, iff there exists a sequence of pairwise distinct prime intervals $\mathfrak{p}=\mathfrak{r}_{0}, \mathfrak{r}_{1}, \ldots, \mathfrak{r}_{n}=\mathfrak{q}$ satisfying

$$
\mathfrak{p}=\mathfrak{r}_{0} \stackrel{p}{\longrightarrow} \mathfrak{r}_{1} \stackrel{p}{\longrightarrow} \cdots \stackrel{p}{\longrightarrow} \mathfrak{r}_{n}=\mathfrak{q} .
$$

\section{The Swing Lemma and the General Swing Lemma}

Now we state the main result in G. Grätzer [22]. For a second proof, see G. Czédli [2] and for the shortest proof, G. Czédli and G. Makay [6].

Lemma 6 (Swing Lemma). Let $L$ be a slim, planar, semimodular lattice and let $\mathfrak{p}, \mathfrak{q}$ be prime intervals in L. Then $\mathfrak{p}$ congruence spreads to $\mathfrak{q}$ iff $\mathfrak{p}$ is SPS projective to $\mathfrak{q}$.

Next we define the concept of switching.

Definition 3. Let $L$ be any finite lattice, and let $\mathfrak{p}, \mathfrak{q}$ be distinct prime intervals in $L$. We say that $\mathfrak{p}$ switches to $\mathfrak{q}$ if both $\mathfrak{p}$ and $\mathfrak{q}$ lie in a common covering $\mathrm{M}_{3}$ in $L$.
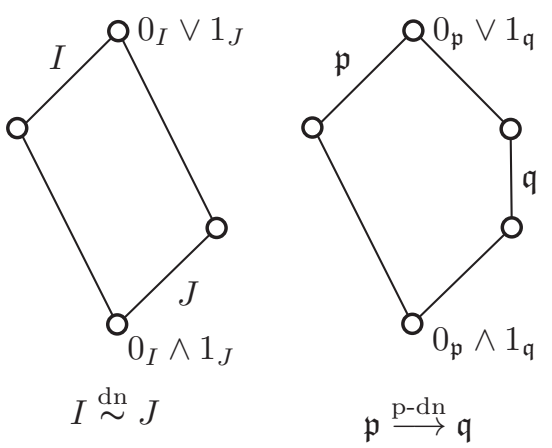

FigURE 3. Introducing prime-perspectivity 
Observe that switching is a symmetric concept, and, furthermore, we have the immediate result:

Lemma 7. Let $L$ be a lattice. If the prime interval $\mathfrak{p}$ switches to the prime interval $\mathfrak{q}$ in $L$, then $\operatorname{con}_{L}(\mathfrak{p})=\operatorname{con}_{L}(\mathfrak{q})$.

We now generalize the Swing Lemma to PS lattices.

Lemma 8 (General Swing Lemma). Let $L$ be a planar semimodular lattice, and let $\mathfrak{p}, \mathfrak{q}$ be distinct prime intervals in L. Then $\mathfrak{q}$ is collapsed by $\operatorname{con}(\mathfrak{p})$ iff there exist sequences of prime intervals in $L, \mathfrak{p}_{0}=\mathfrak{p}, \mathfrak{p}_{1}, \ldots, \mathfrak{p}_{n}$ and $\mathfrak{q}_{0}, \mathfrak{q}_{1}, \ldots, \mathfrak{q}_{n}=\mathfrak{q}$ where $\mathfrak{p}_{k}$ is SPS projective to $\mathfrak{q}_{k}$ for $k=0, \ldots, n$, and, provided that $n>0, \mathfrak{q}_{k}$ switches to $\mathfrak{p}_{k+1}$ for $k=0, \ldots, n-1$.

Since there is no switch in a slim lattice, the Swing Lemma is the General Swing Lemma for slim lattices.

\section{A short approach through planar lattices}

Let $L$ be a planar lattice with an associated planar diagram. A 4-cell $S=\left\{0_{S}, v, w, 1_{S}\right\}$ in $L$ is a covering square with no internal element. We insert an eye $e$ into $S$ by adding an element $e$ to $L$, turning the covering square $S$ into an $\mathrm{M}_{3}$ so that the new element $e$ becomes an internal element of $\left[0_{S}, 1_{S}\right]$ in the lattice $L \cup\{e\}$, denoted by $L^{\mathrm{e}}$.

A quasiordered set $(H, \nu)$ is a nonempty set $H$ with a binary relation $\nu$ that is reflexive and transitive. Following G. Czédli [1], a quasi-coloring of a lattice $M$ of finite length is a surjective map $\gamma$ from $\operatorname{Prime}(M)$ onto a quasiordered set $(H, \nu)$ such that for $\mathfrak{a}, \mathfrak{b} \in \operatorname{Prime}(M)$, the following two conditions hold:

$(\mathrm{C} 1)$ if $(\gamma \mathfrak{a}, \gamma \mathfrak{b}) \in \nu$, then $\mathfrak{b}$ congruence spreads to $\mathfrak{a}$;

$(\mathrm{C} 2)$ if $\mathfrak{b}$ congruence spreads to $\mathfrak{a}$, then $(\gamma \mathfrak{a}, \gamma \mathfrak{b}) \in \nu$.

For $\rho \subseteq Q^{2}$, there is a least quasiorder $\bar{\varrho}$ containing $\rho$ on $Q$; it is the reflexive and transitive extension of $\rho$ on $Q$. Let $(Q, \nu)=\left(\operatorname{Con}_{\mathrm{J}} L, \subseteq\right)$, and let $\gamma$ : Prime $(L) \rightarrow(Q, \nu)$ be the natural quasi-coloring of $L$ defined by the rule $\gamma \mathfrak{a}=\operatorname{con}_{L}(\mathfrak{a})$; see [1, above Lemma 2.1]. Let $\boldsymbol{\alpha}=\gamma\left[v, 1_{S}\right]=\operatorname{con}_{L}\left(v, 1_{S}\right)$ and $\boldsymbol{\beta}=\gamma\left[w, 1_{S}\right]=\operatorname{con}_{L}\left(w, 1_{S}\right)$. Define $\tau$ as the least quasiorder containing $\nu \cup\{(\boldsymbol{\alpha}, \boldsymbol{\beta}),(\boldsymbol{\beta}, \boldsymbol{\alpha})\}$, and extend $\gamma$ to $\gamma^{\mathrm{e}}: \operatorname{Prime}\left(L^{\mathrm{e}}\right) \rightarrow(Q, \tau)$ by $\gamma\left[0_{S}, e\right]=$ $\gamma\left[e, 1_{S}\right]=\boldsymbol{\alpha}$. (G. Czédli [1] uses the notation $L^{\odot}$ and $\gamma^{\odot}$.)

Now we can state the crucial technical lemma G. Czédli [1, Lemma 5.2].

Lemma 9 (Eye Lemma). The map $\gamma^{\mathrm{e}}$ is a quasi-coloring of $L^{\mathrm{e}}$.

Let $L$ be a planar semimodular lattice and let $\mathfrak{p}, \mathfrak{q}$ be distinct prime intervals in $L$. Let us call the sequence provided for $\mathfrak{p}, \mathfrak{q}$ in the General Swing Lemma a $P S$ sequence. If we assume, in addition, that every perspectivity is a perspectivity in a 4-cell, we call the sequence a $P S$ cell sequence ( $\mathrm{PS}^{\mathrm{c}}$ sequence, for short). More exactly, in a planar semimodular lattice $L$, for the prime intervals $\mathfrak{p}$ and $\mathfrak{q}$ in $L$, a PS ${ }^{\mathfrak{c}}$ sequence from $\mathfrak{p}$ to $\mathfrak{q}$ is a sequence $\mathfrak{r}_{0}=\mathfrak{p}, \mathfrak{r}_{1}, \ldots, \mathfrak{r}_{n-1}, \mathfrak{r}_{n}=\mathfrak{q}$ 
of prime intervals of $L$ such that, for each $1 \leq i \leq n$, one of the following three conditions holds.

(i) $\mathfrak{p}_{i-1}$ and $\mathfrak{p}_{i}$ are cell perspective, that is, they are opposite sides of the same 4-cell;

(ii) $\mathfrak{p}_{i-1}$ swings to $\mathfrak{p}_{i}$;

(iii) $\mathfrak{p}_{i-1}$ switches to $\mathfrak{p}_{i}$.

The following statement is well known.

Lemma 10. Let $L$ be a planar lattice and let $S=\left\{0_{S}, v, w, 1_{S}\right\}$ be a 4-cell in $L$. If we insert an eye e into $S$, then any two prime intervals of $S \cup\{e\}=\mathrm{M}_{3}$ are connected with a $P S^{\mathrm{c}}$ sequence.

We are now ready to prove the General Swing Lemma in the following equivalent form.

Lemma 11 (General Swing Lemma'). Let $L$ be a planar semimodular lattice and let $\mathfrak{p}, \mathfrak{q}$ be distinct prime intervals in $L$. Then $\mathfrak{p}$ congruence spreads to $\mathfrak{q}$ iff there exists a $P S^{\mathrm{c}}$ sequence from $\mathfrak{p}$ to $\mathfrak{q}$.

Proof. The sufficiency is straightforward by Lemma 3 .

We prove the necessity by induction on the number of eyes in $L$. If this number is 0 , then $L$ is slim, so the statement follows from the Swing Lemma.

So assume the validity of the lemma for a planar semimodular lattice $L$ and insert an eye $e$ into a 4-cell $S=\left\{0_{S}, v, w, 1_{S}\right\}$. Let $M=S \cup\{e\}$. We want to prove that the General Swing Lemma' holds in $L^{\mathrm{e}}$. To accomplish this assume that $\mathfrak{p}, \mathfrak{q}$ are prime intervals of $L^{\mathrm{e}}$ and $\mathfrak{p}$ congruence spreads to $\mathfrak{q}$. We have to find a $\mathrm{PS}^{\mathrm{c}}$ sequence from $\mathfrak{p}$ to $\mathfrak{q}$.

Let us assume that

$$
\mathfrak{p}, \mathfrak{q} \subseteq L .
$$

By the Eye Lemma, $\gamma^{\mathrm{e}}$ is a quasi-coloring. Since $\mathfrak{p}$ congruence spreads to $\mathfrak{q}$ in $L^{\mathrm{e}},(\mathrm{C} 2)$ yields that $\left(\gamma^{\mathrm{e}} \mathfrak{q}, \gamma^{\mathrm{e}} \mathfrak{p}\right) \in \tau$. Hence, there is a sequence $\boldsymbol{\delta}_{0}=\gamma^{\mathrm{e}} \mathfrak{q}$, $\boldsymbol{\delta}_{1}, \ldots, \boldsymbol{\delta}_{n-1}, \boldsymbol{\delta}_{n}=\gamma^{\mathrm{e}} \mathfrak{p}$ of elements of $Q=\operatorname{Con}_{\mathrm{J}} L$ such that $\left(\boldsymbol{\delta}_{i-1}, \boldsymbol{\delta}_{i}\right) \in$ $\{(\boldsymbol{\alpha}, \boldsymbol{\beta}),(\boldsymbol{\beta}, \boldsymbol{\alpha})\} \cup \nu$ for $i \in\{1, \ldots, n\}$. Let $\mathfrak{r}_{0}=\mathfrak{q}$ and $\mathfrak{r}_{n}=\mathfrak{p}$. For each $i \in\{1, \ldots, n-1\}$, the surjectivity of $\gamma$ allows us to pick a prime interval $\mathfrak{r}_{i} \in \operatorname{Prime}(L)$ such that $\gamma \mathfrak{r}_{i}=\boldsymbol{\delta}_{i}$; note that this equality holds also for $i \in\{0, n\}$, since $\mathfrak{p}, \mathfrak{q} \in \operatorname{Prime}(L)$ and $\gamma^{\mathrm{e}}$ extends $\gamma$. For $i \in\{1, \ldots, n\}$, there are two cases.

First, assume that $\left(\boldsymbol{\delta}_{i-1}, \boldsymbol{\delta}_{i}\right) \in \nu$. Since $\left(\gamma \mathfrak{r}_{i-1}, \gamma \mathfrak{r}_{i}\right)=\left(\boldsymbol{\delta}_{i-1}, \boldsymbol{\delta}_{i}\right) \in \nu,(\mathrm{C} 1)$ gives that $\operatorname{con}_{L}\left(\mathfrak{r}_{i-1}\right) \subseteq \operatorname{con}_{L}\left(\mathfrak{r}_{i}\right)$, whereby the induction hypothesis yields a PS sequence $\vec{s}_{i}{ }^{\prime}$ from $\mathfrak{r}_{i}$ to $\mathfrak{r}_{i-1}$ in $L$. If two consecutive members of $\vec{s}_{i}{ }^{\prime}$ belong to Prime $(S)$, then $\vec{s}_{i}{ }^{\prime}$ is not a $\mathrm{PS}^{\mathrm{c}}$ sequence in $L^{\mathrm{e}}$, but Lemma 10 helps in turning it into a PS ${ }^{\mathrm{c}}$ sequence $\vec{s}_{i}$ from $\mathfrak{r}_{i}$ to $\mathfrak{r}_{i-1}$ in $L^{\mathrm{e}}$.

Second, assume that $\left(\boldsymbol{\delta}_{i-1}, \boldsymbol{\delta}_{i}\right)=(\boldsymbol{\alpha}, \boldsymbol{\beta})$ or $(\boldsymbol{\beta}, \boldsymbol{\alpha})$. By reason of symmetry, we can assume that $\left(\boldsymbol{\delta}_{i-1}, \boldsymbol{\delta}_{i}\right)=(\boldsymbol{\alpha}, \boldsymbol{\beta})$. Since $\gamma\left(\mathfrak{r}_{i-1}\right)=\boldsymbol{\delta}_{i-1}=\boldsymbol{\alpha}=\gamma\left[v, 1_{S}\right]$, it follows that $\operatorname{con}_{L}\left(\mathfrak{r}_{i-1}\right) \subseteq \operatorname{con}_{L}\left(v, 1_{S}\right)$ by $(\mathrm{C} 1)$. As in the first case, the 
induction hypothesis and Lemma 10 yield a $\mathrm{PS}^{\mathrm{c}}$ sequence $\vec{s}_{i}{ }^{\prime}$ from $\left[v, 1_{S}\right]$ to $\mathfrak{r}_{i-1}$ in $L^{\mathrm{e}}$. Similarly, we have a $\mathrm{PS}^{\mathrm{c}}$ sequence $\vec{s}_{i}^{\prime \prime}$ from $\mathfrak{r}_{i}$ to $\left[w, 1_{S}\right]$ in $L^{\mathrm{e}}$. Since Lemma 10 gives a $\mathrm{PS}^{\mathrm{c}}$ sequence $\vec{s}_{i}{ }^{\prime \prime \prime}$ from $\left[w, 1_{S}\right]$ to $\left[v, 1_{S}\right]$ in $L^{\mathrm{e}}$, the concatenation of $\vec{s}_{i}{ }^{\prime \prime}, \vec{s}_{i}{ }^{\prime \prime \prime}$, and $\vec{s}_{i}{ }^{\prime}$ is a PS sequence $\vec{s}_{i}$ from $\mathfrak{r}_{i}$ to $\mathfrak{r}_{i-1}$ in $L^{\mathrm{e}}$.

Finally, the concatenation of $\vec{s}_{n}, \vec{s}_{n-1}, \ldots, \vec{s}_{1}$ is a PS' sequence from $\mathfrak{p}$ to $\mathfrak{q}$ in $L^{\mathrm{e}}$, as required.

It remains to prove this lemma if (3) fails. In this case, we have that $e \in \mathfrak{p} \cup \mathfrak{q}$. If $e \in \mathfrak{p}$, then $\mathfrak{p} \in \operatorname{Prime}(M)$. Let $\mathfrak{p}^{\prime}$ be any prime interval in $S \subseteq L$. Then $\operatorname{con}\left(\mathfrak{p}^{\prime}\right)=\operatorname{con}(\mathfrak{p})$ in $L^{\mathrm{e}}$ and, by Lemma 10 , there is a PS' sequence from $\mathfrak{p}$ to $\mathfrak{p}^{\prime}$ in $L^{\mathrm{e}}$. If $e \notin \mathfrak{p}$, just set $\mathfrak{p}^{\prime}=\mathfrak{p}$. Similarly, if $e \in \mathfrak{q}$, then, for any prime interval $\mathfrak{q}^{\prime}$ in $S, \operatorname{con}\left(\mathfrak{q}^{\prime}\right)=\operatorname{con}(\mathfrak{q})$ and there is a PS ${ }^{c}$ sequence from $\mathfrak{q}^{\prime}$ to $\mathfrak{q}$ in $L^{\mathrm{e}}$. Otherwise, set $\mathfrak{q}^{\prime}=\mathfrak{q}$. Then $\mathfrak{p}^{\prime}$ congruence spreads to $\mathfrak{q}^{\prime}$ in $L^{\mathrm{e}}$ and $\mathfrak{p}^{\prime}, \mathfrak{q}^{\prime}$ satisfy (3). Thus there is a PSc sequence from $\mathfrak{p}^{\prime}$ to $\mathfrak{q}^{\prime}$ in $L^{\mathrm{e}}$. Combining these $\mathrm{PS}^{\mathrm{c}}$ sequences, we get a $\mathrm{PS}^{\mathrm{c}}$ sequence in $L^{\mathrm{e}}$ from $\mathfrak{p}$ to $\mathfrak{q}$.

\section{The Tab Lemma for finite lattices}

We now state our main technical lemma for finite lattices.

Lemma 12 (Tab Lemma). Let $L$ be a finite lattice, and let $m$ be a tab of $L$ in the covering multi-diamond $[o, i]$. Let $K=L-\{m\}$, a sublattice of $L$. Let $\boldsymbol{\alpha} \in \operatorname{Con} K$ and let $\boldsymbol{\beta}=\operatorname{con}_{L}(\boldsymbol{\alpha})$. Then

$$
\boldsymbol{\beta}\rceil K= \begin{cases}\boldsymbol{\alpha}, & \text { if } o \neq \equiv i \quad(\bmod \boldsymbol{\beta}) ; \\ \boldsymbol{\alpha} \vee_{K} \operatorname{con}_{K}(o, i), & \text { otherwise. }\end{cases}
$$

Note that the first case means that $\boldsymbol{\beta}$ is an extension of $\boldsymbol{\alpha}$.

Proof. We first consider the case $o \not \equiv i(\bmod \boldsymbol{\beta})$. We extend the congruence relation $\boldsymbol{\alpha}$ of $K$ to the equivalence relation $\gamma$ of $L$ by making the equivalence class of $m$ the singleton $\{m\}$. We observe that we cannot have $x<m<y$ for some $x, y \in K$ with $x \equiv y(\bmod \boldsymbol{\alpha})$; indeed, $x \leq m_{*}<m<m^{*} \leq y$, so, since $o=m_{*}$ and $i=m^{*}$, we would have $o \equiv i(\bmod \boldsymbol{\alpha})$ and thereby $o \equiv i$ $(\bmod \boldsymbol{\beta})$.

Thus all the equivalence classes of $\gamma$ are intervals in $L$ (the intervals in $K$ and $\{m\})$ and we can apply Lemma 4 to show that $\gamma$ is a congruence relation on $L$.

Let $x, y, z$ be distinct elements of $L$ with $y, z$ covering $x$ and $x \equiv y(\bmod \gamma)$. Since the equivalence class of $m$ is a singleton, it follows that $x \neq m$ and $y \neq m$. But $z=m$ is also impossible. Indeed, $x \equiv y(\bmod \boldsymbol{\alpha})$ and so $x \equiv y(\bmod \boldsymbol{\beta})$ and $\boldsymbol{\beta}$ is a congruence relation on $L$, whereby $y \vee z \equiv z(\bmod \boldsymbol{\beta})$, and $y \vee z>z$. Since $m^{*}=i$, from $z=m$ it would follow that $i \equiv m(\bmod \boldsymbol{\beta})$, that is, $o \equiv i$ $(\bmod \boldsymbol{\beta})$, contradicting our hypothesis that $o \not \equiv i(\bmod \boldsymbol{\beta})$ - see Figure 4.

Consequently, $x, y, z$ are all elements of $K$, and $x \equiv y(\bmod \boldsymbol{\alpha})$. Then $z \equiv y \vee z(\bmod \boldsymbol{\alpha})$, that is $z \equiv y \vee z(\bmod \gamma)$. This argument and its dual show that $\gamma$ is a congruence relation on $L$. 
Then, it is immediate from its definition that $\boldsymbol{\gamma}=\operatorname{con}_{L}(\boldsymbol{\alpha})=\boldsymbol{\beta}$ and $\boldsymbol{\beta}\rceil K=\boldsymbol{\alpha}$.

It remains to consider the case $o \equiv i(\bmod \boldsymbol{\beta})$. Let us denote the congruence relation $\boldsymbol{\alpha} \vee_{K} \operatorname{con}_{K}(o, i)$ of $K$ by $\boldsymbol{\alpha}^{\prime}$. We extend $\boldsymbol{\alpha}^{\prime}$ to an equivalence relation $\boldsymbol{\gamma}$ on $L$ by setting $x \equiv y(\bmod \gamma)$ for all $x, y \in[o, i]$. Since $o=m_{*}$ and $i=m^{*}$, and $o \equiv i\left(\bmod \boldsymbol{\alpha}^{\prime}\right)$ it follows that all equivalence classes of $\boldsymbol{\gamma}$ are intervals in $L$. We then apply Lemma 4 to $\gamma$ to show that $\gamma$ is a congruence relation on $L$.

Let $x, y, z$ be distinct elements of $L$ with $y, z$ covering $x$ and $x \equiv y(\bmod \gamma)$.

If $x, y, z$ are all elements of $K$, then $x \equiv y\left(\bmod \boldsymbol{\alpha}^{\prime}\right)$ and so $y \equiv y \vee z$ $\left(\bmod \boldsymbol{\alpha}^{\prime}\right)$, that is $y \equiv y \vee z(\bmod \gamma)$.

The case $x=m$ is impossible, since $m$ has only one upper cover.

The case $y=m$ implies that $z \in K$ and that $o=x<z$, since $o=m_{*}$. Since $y \vee z>y=m$, we have that $y \vee z \geq i>y$. Thus

$$
y \vee z=i \vee z \equiv o \vee z=z \quad\left(\bmod \boldsymbol{\alpha}^{\prime}\right),
$$

that is, $y \vee z \equiv z(\bmod \gamma)$-see Figure 5 .

The case $z=m$ implies that $y \in K$ and $x=o$. Thus $y \equiv o\left(\bmod \boldsymbol{\alpha}^{\prime}\right)$. Furthermore, $y \vee z \geq i$ since $y \vee z>z=m$, whereby

$$
y \vee z=y \vee i \equiv o \vee i=i \quad\left(\bmod \boldsymbol{\alpha}^{\prime}\right),
$$

that is, $y \vee z \equiv i(\bmod \gamma)$-see Figure 6 . But $i \equiv m(\bmod \gamma)$ by the definition of $\gamma$. Consequently, $y \vee z \equiv m=z(\bmod \gamma)$ since $\gamma$ is an equivalence relation.

Thus by the above argument and its dual, $\gamma$ is a congruence relation on $L$.

Now $\boldsymbol{\alpha} \subseteq \boldsymbol{\gamma}$. Thus $\boldsymbol{\beta}=\operatorname{con}_{L}(\boldsymbol{\alpha}) \leq \boldsymbol{\gamma}$. Since, clearly,

$$
\gamma\rceil K=\boldsymbol{\alpha}^{\prime}=\boldsymbol{\alpha} \vee_{K} \operatorname{con}_{K}(o, i),
$$

it follows that $\boldsymbol{\beta}\rceil K=\boldsymbol{\alpha}^{\prime}=\boldsymbol{\alpha} \vee_{K} \operatorname{con}_{K}(o, i)$.

Now we shall discuss some applications of the Tab Lemma. In these discussions, $L$ is a finite lattice, $m$ is a tab in a covering multi-diamond $[o, i]$ of $L$, and the sublattice $K=L-\{m\}$.

We first make a very simple observation.

Lemma 13. Let $\mathfrak{p}$ and $\mathfrak{q}$ be prime intervals in $K$. If $\mathfrak{p}$ is prime-perspective down to $\mathfrak{q}$ in $L$, then $\mathfrak{p}$ is prime-perspective down to $\mathfrak{q}$ in $K$, and dually for prime-perspective up.

Proof. Clearly, we may assume that $\mathfrak{p}$ and $\mathfrak{q}$ are distinct intervals. Now the end-points $0_{\mathfrak{p}}, 1_{\mathfrak{p}}, 0_{\mathfrak{q}}, 1_{\mathfrak{q}}$ are all distinct from $m$. There is at most one other element involved, which is $0_{\mathfrak{p}} \wedge 1_{\mathfrak{q}}$, which is meet-reducible, and so is also distinct from $m$. Thus the prime-perspectivity occurs in $K$.

Lemma 14. Let $\mathfrak{p}$ be a prime interval in $K$. Then $o \equiv i\left(\bmod \operatorname{con}_{L}(\mathfrak{p})\right)$ iff there is a prime interval $\mathfrak{q}$ in $K$ with $0_{\mathfrak{q}} \equiv 1_{\mathfrak{q}}\left(\bmod \operatorname{con}_{K}(\mathfrak{p})\right)$ and either $1_{\mathfrak{q}}=i$ or, dually, $0_{\mathfrak{q}}=o$. 


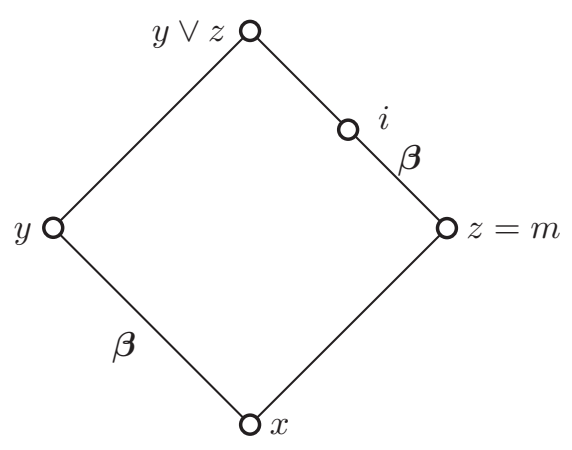

Figure 4. Case $o \not \equiv i(\bmod \boldsymbol{\beta})$ and $z=m$ of Lemma 12

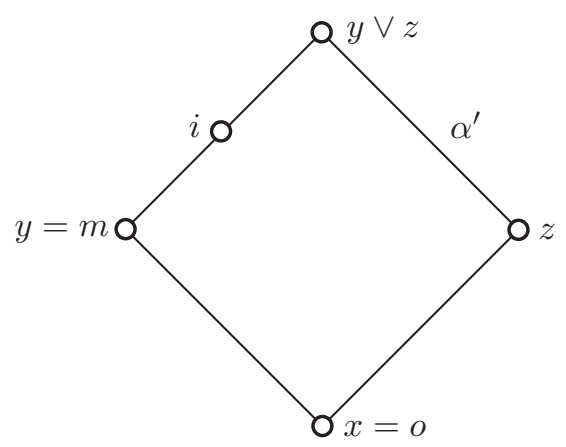

Figure 5 . Case $o \equiv i(\bmod \boldsymbol{\beta})$ and $y=m$ of Lemma 12

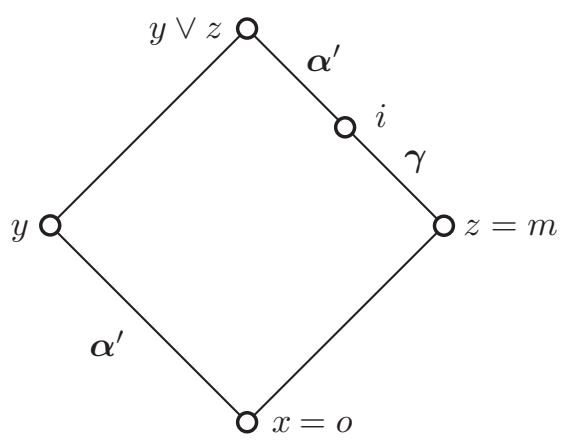

FiguRe $6 . o \equiv i(\bmod \boldsymbol{\beta})$ and $z=m$ case of Lemma 12

Proof. First, assume that there is such a prime interval $\mathfrak{q}$ with $1_{\mathfrak{q}}=i$. Now $0_{\mathfrak{q}} \neq m$ and so $0_{\mathfrak{q}} \vee m=1_{\mathfrak{q}}$. Furthermore, $0_{\mathfrak{q}} \wedge m<m$. Since $m_{*}=o$ in $L$, we have $0_{\mathfrak{q}} \wedge m \leq o$, that is, the prime interval $\mathfrak{q}$ is prime-perspective down to the prime interval $[o, m]$. Since $0_{\mathfrak{q}} \equiv 1_{\mathfrak{q}}\left(\bmod \operatorname{con}_{L}(\mathfrak{p})\right)$, we conclude that 
$o \equiv m\left(\bmod \operatorname{con}_{L}(\mathfrak{p})\right)$. Since $[o, i]$ is a simple sublattice of $L$, it follows that $o \equiv i\left(\bmod \operatorname{con}_{L}(\mathfrak{p})\right)$, proving one direction of the equivalence.

Second, assume that $o \equiv i\left(\bmod \operatorname{con}_{L}(\mathfrak{p})\right)$. Then $m \equiv i\left(\bmod \operatorname{con}_{L}(\mathfrak{p})\right)$. Then there is a prime-projectivity, a sequence of prime-perspectivities starting at $\mathfrak{p}$ and ending at the prime interval $[m, i]$. In this sequence, there is a first prime interval $\mathfrak{r}^{\prime}$ containing $m$-it is not $\mathfrak{p}$, since $\mathfrak{p} \subseteq K$. Thus there is an immediate previous prime interval $\mathfrak{r}$ in this sequence, which by duality, we may assume is prime-perspective down to $\mathfrak{r}^{\prime}$. By the choice of $\mathfrak{r}^{\prime}, 0_{\mathfrak{r}}, 1_{\mathfrak{r}} \in K$ and, by Lemma 13,

$$
0_{\mathfrak{r}} \equiv 1_{\mathfrak{r}} \quad\left(\bmod \operatorname{con}_{K}(\mathfrak{p})\right) .
$$

The element $m$ is doubly-irreducible, so it occurs in only two prime intervals of $L$, the prime interval $[m, i]$ and the prime interval $[o, m]$. So either $\mathfrak{r}^{\prime}=[m, i]$ or $\mathfrak{r}^{\prime}=[o, m]$.

We first consider the case $\mathfrak{r}^{\prime}=[m, i]$. Then $0_{\mathfrak{r}} \wedge i \leq m$. Since $m$ is meetirreducible and $m_{*}=o$, we get that $0_{\mathfrak{r}} \wedge i \leq o$. But then $\mathfrak{r}$ is prime-projective down to $[a, i]$, where $a$ is any of the other atoms of the covering multi-diamond $[o, i]$ - see Figure 7. So we can take $\mathfrak{q}=[a, i]$. Observe that, in this case, qis actually an interval in $[o, i]$.

We are left with the case $\mathfrak{r}^{\prime}=[o, m]$. Since $m \notin \mathfrak{r}$, it follows that $1_{\mathfrak{r}}>m$, and so $1_{\mathfrak{r}} \geq i=m^{*}$. Also, $0_{\mathfrak{r}} \wedge i<i$, since $0_{\mathfrak{r}} \wedge m \leq o<m$. Let $b$ be a lower cover of $i$ in the interval $\left[0_{\mathfrak{r}} \wedge i, i\right]$. By $(4), 0_{\mathfrak{r}} \wedge i \equiv i\left(\bmod \operatorname{con}_{K}(\mathfrak{p})\right)$, and so $b \equiv i\left(\bmod \operatorname{con}_{K}(\mathfrak{p})\right)$-see Figure 8 . We then set $\mathfrak{q}=[b, i]$, concluding the proof. Observe that in this case, $\mathfrak{q}$ need not be in $[o, i]$ since $b$ need not be $\geq 0$.

If $L$ is semimodular, then we need not worry about the dual possibility.

Lemma 15. Let us further assume that $L$ is semimodular. Let $\mathfrak{p}$ be a prime interval in $K$. Then $o \equiv i\left(\bmod \operatorname{con}_{L}(\mathfrak{p})\right)$ iff there is a prime interval $\mathfrak{q}$ in $K$ with $0_{\mathfrak{q}} \equiv 1_{\mathfrak{q}}\left(\bmod \operatorname{con}_{K}(\mathfrak{p})\right)$ and $1_{\mathfrak{q}}=i$.

If $0_{\mathfrak{q}} \notin[o, i]$, then $\mathfrak{q}$ swings in $L$ to some prime interval $\mathfrak{r}$ in $[o, i]-\{m\}$.

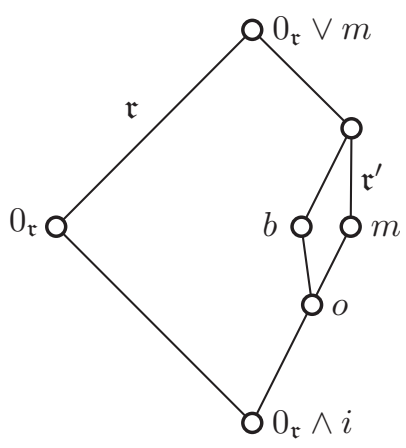

FiguRE $7 \cdot \mathfrak{r}^{\prime}=[m, i]$ case of Lemma 14 


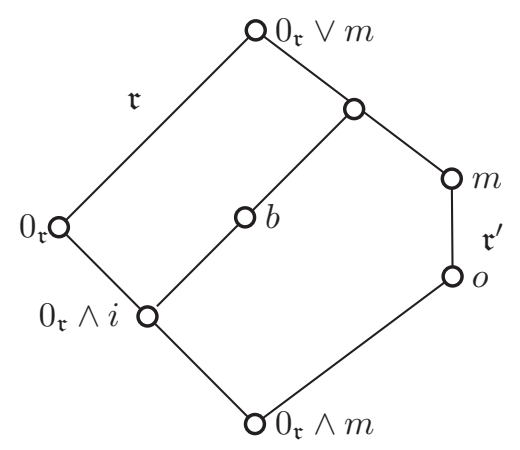

FiguRE $8 . \mathfrak{r}^{\prime}=[o, m]$ case of Lemma 14

Proof. We first consider the case when there is a prime interval $\mathfrak{q}^{\prime}$ in $K$ with

$$
0_{\mathfrak{q}^{\prime}} \equiv 1_{\mathfrak{q}^{\prime}} \quad\left(\bmod \operatorname{con}_{K}(\mathfrak{p})\right)
$$

and $0_{\mathfrak{q}^{\prime}}=o$. Now $1_{\mathfrak{q}^{\prime}}$ is distinct from $m$. By semimodularity, $1_{\mathfrak{q}^{\prime}} \vee m$ covers $m$ and $1_{\mathfrak{q}^{\prime}}$. So $1_{\mathfrak{q}^{\prime}} \vee m=i=m^{*}$. Then

$$
i=1_{\mathfrak{q}^{\prime}} \vee m \equiv 1_{\mathfrak{q}^{\prime}} \quad\left(\bmod \operatorname{con}_{K}(\mathfrak{p})\right) .
$$

Setting $\mathfrak{q}=\left[1_{\mathfrak{q}^{\prime}}, i\right]$ and noting that then $0_{\mathfrak{q}}=1_{\mathfrak{q}^{\prime}} \in[o, i]$, completes the proof in this case.

Otherwise, by Lemma 14, there is a prime interval $\mathfrak{q}$ in $K$ with

$$
0_{\mathfrak{q}} \equiv 1_{\mathfrak{q}} \quad\left(\bmod \operatorname{con}_{K}(\mathfrak{p})\right)
$$

and with $1_{\mathfrak{q}}=i$. If $0_{\mathfrak{q}} \notin[o, i]$, then we apply Lemma 2 , with $x=0_{\mathfrak{q}}$, to get the prime interval $\mathfrak{r}=[a, i]$ in $[o, i]-\{m\}$, where $\mathfrak{q}$ swings to $\mathfrak{r}$ by use of $m$ (and thus the swing is in $L$, but not necessarily in $K$ ).

\section{The proof of the General Swing Lemma}

Our proof of the General Swing Lemma for a planar semimodular lattice will be by induction on the number of tabs in the lattice. We then need the following very easy result.

Lemma 16. Let $L$ be a finite semimodular lattice containing a doubly-irreducible element $m$. Then the sublattice $K=L-\{m\}$ is also semimodular.

Proof. Let $a, b, c \in K$ with $b \neq c$ covering $a$. We have to show that $b$ and $c$ cover $a$ in $L$; see G. Grätzer [11, Theorem 375].

Assume to the contrary that $b$ does not cover $a$ in $L$. Then $a \prec m \prec b$ in $L$. So $m$ and $c$ are distinct upper covers of $a$. But $L$ is semimodular, so $m \vee c$ covers both $m$ and $c$ - see Figure 9. Since it is meet-irreducible, $m$ has only one upper cover, and so $m \vee c=b$. Thus, $b$ covers $c$, that is, $a \prec c \prec b$ in $K$, contradicting $a \prec b$ in $K$. 


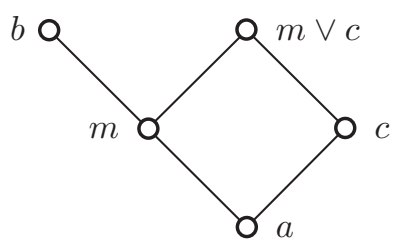

Figure 9. Lemma 16: $a \prec m \prec b$

Thus, $b$ and $c$ still cover $a$ in $L$. Then, by the semimodularity of $L, b \vee c$ covers $b$ and $c$ in $L$ and so, certainly, in $K$. Consequently, $K$ is semimodular.

We now prove the General Swing Lemma.

Proof of Lemma 8. First, let $\mathfrak{p}, \mathfrak{q}$ be prime intervals in $L$, and let there be such sequences $\mathfrak{p}_{0}=\mathfrak{p}, \mathfrak{p}_{1}, \ldots, \mathfrak{p}_{n}$ and $\mathfrak{q}_{0}, \mathfrak{q}_{1}, \ldots, \mathfrak{q}_{n}=\mathfrak{q}$. By Lemma 3 , each $\mathfrak{q}_{k}$ is collapsed by $\operatorname{con}\left(\mathfrak{p}_{k}\right)$, and, by Lemma 7 , each $\mathfrak{p}_{k+1}$ is collapsed by $\operatorname{con}\left(\mathfrak{q}_{k}\right)$. Then $\mathfrak{q}$ is collapsed by $\operatorname{con}(\mathfrak{p})$.

We now proceed in the other direction. Let $\mathfrak{p}$ and $\mathfrak{q}$ be distinct prime intervals in $L$, and let $\mathfrak{q}$ be collapsed by $\operatorname{con}(\mathfrak{p})$. We proceed by induction on the number of tabs of $L$.

Note that in a covering multi-diamond in any planar lattice $L^{\prime}$ each atom, except possibly the leftmost and rightmost, is doubly-irreducible in $L^{\prime}$, that is, it is a tab of $L^{\prime}$. So if there are no tabs in the lattice $L$, then $L$ has no covering multi-diamond, that is, is slim. So by the Swing Lemma, $\mathfrak{p}$ is SPS projective to $\mathfrak{q}$. We then get the conclusion if there are no tabs.

Now let there be at least one tab in $L$. Take such a tab $m$, an atom of a covering multi-diamond $[o, i]$ in $L$, and set $K=L-\{m\}$. Then, by Lemma 16, $K$ is a planar semimodular lattice, and, by the induction hypothesis, the General Swing Lemma holds for the lattice $K$.

If $m \in \mathfrak{p}$, then let $\mathfrak{p}^{\prime}$ be a prime interval in $[o, i]$ that does not contain $m$. Then by definition, $\mathfrak{p}$ switches to $\mathfrak{p}^{\prime}$, and, since $\operatorname{con}_{L}(\mathfrak{p})=\operatorname{con}_{L}\left(\mathfrak{p}^{\prime}\right)$, the prime interval $\mathfrak{q}$ is collapsed by $\operatorname{con}_{L}\left(\mathfrak{p}^{\prime}\right)$. Similarly, if $m \in \mathfrak{q}$, then there is a prime interval $\mathfrak{q}^{\prime} \subseteq[o, i]$ that switches to $\mathfrak{q}$, that does not contain $m$, but is collapsed by $\mathfrak{p}$.

Thus to prove the General Swing Lemma, it suffices to assume that $m$ is an element of neither $\mathfrak{p}$ nor $\mathfrak{q}$, that is, that $\mathfrak{p}$ and $\mathfrak{q}$ lie in $K$. If $\mathfrak{q}$ is collapsed by $\operatorname{con}_{K}(\mathfrak{p})$, then we are done, since $K$ satisfies the General Swing Lemma.

Otherwise, since $\mathfrak{q}$ is collapsed by $\operatorname{con}_{L}(\mathfrak{p})$, it follows that $\left.\operatorname{con}_{L}(\mathfrak{p})\right\rceil K \neq$ $\operatorname{con}_{K}(\mathfrak{p})$. Since $\operatorname{con}_{L}(\mathfrak{p})=\operatorname{con}_{L}\left(\operatorname{con}_{K}(\mathfrak{p})\right)$, we conclude by Lemma 12 that

$$
o \equiv i \quad\left(\bmod \operatorname{con}_{L}(\mathfrak{p})\right)
$$

and that

$$
\left.\operatorname{con}_{L}(\mathfrak{p})\right\rceil K=\operatorname{con}_{K}(\mathfrak{p}) \vee_{K} \operatorname{con}_{K}(o, i)
$$


By (5) and Lemma 15, there is a prime interval $\mathfrak{r}$ in $K$ with $1_{\mathfrak{r}}=i$ that is collapsed in $K$ by $\operatorname{con}_{K}(\mathfrak{p})$. Since the General Swing Lemma holds for $K$, there are sequences of prime intervals in $K, \mathfrak{p}_{0}=\mathfrak{p}, \mathfrak{p}_{1}, \ldots, \mathfrak{p}_{n}$ and $\mathfrak{q}_{0}, \mathfrak{q}_{1}, \ldots, \mathfrak{q}_{n}=\mathfrak{r}$, where $\mathfrak{p}_{k}$ is SPS projective to $\mathfrak{q}_{k}$ in $K$ for $k=0, \ldots, n$, and $\mathfrak{q}_{k}$ switches to $\mathfrak{p}_{k+1}$ in $K$ for $k=0, \ldots, n-1$, provided that $n>0$.

If $\mathfrak{r} \nsubseteq[o, i]$, then by Lemma $15, \mathfrak{r}$ swings in $L$ to some prime interval $\mathfrak{r}^{\prime}$ in $[o, i] \cap K$. Then since $\mathfrak{p}_{n}$ is SPS projective in $K$ to $\mathfrak{r}$, it follows that $\mathfrak{p}_{n}$ is SPS projective in $L$ to $\mathfrak{r}^{\prime}$, a prime interval in $[o, i] \cap K$. So if $\mathfrak{r}$ is not in $[o, i]$, we can replace $\mathfrak{r}$ by $\mathfrak{r}^{\prime}$.

In either event, we have sequences of prime intervals in $K, \mathfrak{p}_{0}=\mathfrak{p}, \mathfrak{p}_{1}, \ldots, \mathfrak{p}_{n}$ and $\mathfrak{q}_{0}, \mathfrak{q}_{1}, \ldots, \mathfrak{q}_{n}$ where $\mathfrak{p}_{k}$ is SPS projective to $\mathfrak{q}_{k}$ in $L$ for $k=0, \ldots, n$, and $\mathfrak{q}_{k}$ switches to $\mathfrak{p}_{k+1}$ in $K$ for $k=0, \ldots, n-1$, provided that $n>0$, and where $\mathfrak{q}_{n}$ is an interval in $[o, i] \cap K$.

Since $\mathfrak{q}$ lies in $K$ and is collapsed by $\operatorname{con}_{L}(\mathfrak{p})$, it follows from (6) that $\mathfrak{q}$ is collapsed by $\operatorname{con}_{K}(\mathfrak{p}) \vee_{K} \operatorname{con}_{K}(o, i)$. Choose an atom $a$ of the multi-diamond $[o, i]$ distinct from $m$ and not an element of $\mathfrak{q}_{n}$. Then

$$
\operatorname{con}_{K}(\mathfrak{q}) \leq \operatorname{con}_{K}(\mathfrak{p}) \vee_{K} \operatorname{con}_{K}(o, i)=\operatorname{con}_{K}(\mathfrak{p}) \vee_{K} \operatorname{con}_{K}(o, a) \vee_{K} \operatorname{con}_{K}(a, i) .
$$

(This is the best we can hope for, since $[o, i] \cap K$ need not be simple.) Since $\operatorname{con}_{K}(\mathfrak{q})$ is join-irreducible and is not $\leq \operatorname{con}_{K}(\mathfrak{p})$, we conclude that

$$
\operatorname{con}_{K}(\mathfrak{q}) \leq \operatorname{con}_{K}(\mathfrak{s}) \text { where } \mathfrak{s}=[0, a] \text { or } \mathfrak{s}=[a, i] .
$$

Since $a \notin \mathfrak{q}_{n}$, it follows that $\mathfrak{q}_{n}$ and $\mathfrak{s}$ are distinct prime intervals in $[o, i]$. Thus, $\mathfrak{q}_{n}$ switches in $L$ to $\mathfrak{s}$.

We now proceed to extend the sequences $\mathfrak{p}_{0}, \ldots, \mathfrak{p}_{n}$ and $\mathfrak{q}_{0}, \ldots, \mathfrak{q}_{n}$.

Set $\mathfrak{p}_{n+1}=\mathfrak{s}$. Then $\mathfrak{q}_{n}$ switches in $L$ to $\mathfrak{p}_{n+1}$. By (7), since $K$ satisfies the General Swing Lemma, there are sequences of prime intervals in $K, \mathfrak{p}_{n+1}=$ $\mathfrak{s}, \ldots, \mathfrak{p}_{r}$ and $\mathfrak{q}_{n+1}, \ldots, \mathfrak{q}_{r}=\mathfrak{q}$ where $\mathfrak{p}_{k}$ is SPS projective in $K$ to $\mathfrak{q}_{k}$ for $k=n+1, \ldots, r$ and $\mathfrak{q}_{k}$ switches in $K$ to $\mathfrak{p}_{k+1}$ for $k=n+1, \ldots, r-1$. Combining these sequences, we get the desired sequences $\mathfrak{p}_{0}=\mathfrak{p}, \ldots, \mathfrak{p}_{n+1}, \ldots \mathfrak{p}_{r}$ and $\mathfrak{q}_{0}, \ldots, \mathfrak{q}_{n}, \ldots, \mathfrak{q}_{r}=\mathfrak{q}$, establishing the General Swing Lemma for $L$, and so concluding the proof of the induction step.

Thus the proof of the General Swing Lemma for planar semimodular lattices is concluded.

One should note that, in the General Swing Lemma, some of the SPS projectivities could be trivial - there could be two successive switches in different multi-diamonds, or we could start off with a switch rather than a proper SPSprojectivity.

\section{Discussion}

In the book [16], Section 24.4 discusses some consequences of the Swing Lemma. We take up here only one aspect of this topic. 
Lemma 17. Let $L$ be a planar semimodular lattice, and let $\boldsymbol{\alpha}, \boldsymbol{\beta} \in \operatorname{Con}_{\mathrm{J}} L$ be join-irreducible congruences. If $\boldsymbol{\beta}<\boldsymbol{\alpha}$, then there exist prime intervals $\mathfrak{p}$ and $\mathfrak{q}$ in $L$ such that

(i) $\mathfrak{p}$ swings to $\mathfrak{q}$,

(ii) $\boldsymbol{\beta} \leq \operatorname{con}(\mathfrak{q})<\operatorname{con}(\mathfrak{p}) \leq \boldsymbol{\alpha}$, and

(iii) $\operatorname{con}(\mathfrak{p})$ covers $\operatorname{con}(\mathfrak{q})$ in the order $\left(\operatorname{Con}_{\mathrm{J}} L ; \leq\right)$.

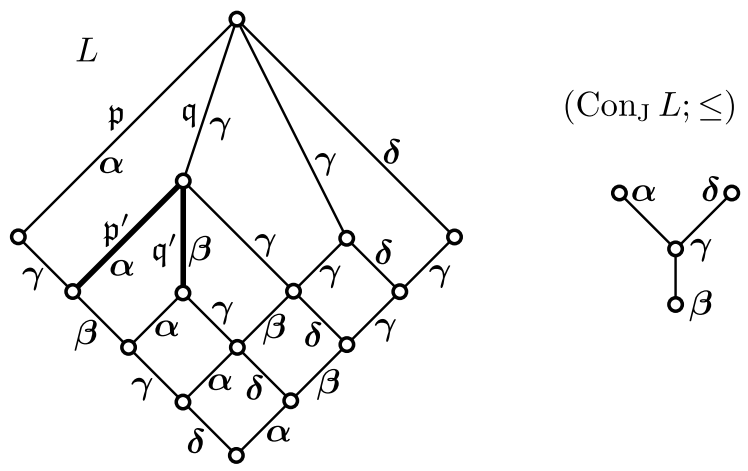

FIGURE 10. A swing need not give covering

Figure 10 shows that this lemma does not follow as immediately from the General Swing Lemma. This figure defines a planar semimodular lattice $L$, which is actually an SPS lattice. Although the Swing Lemma in itself is appropriate to determine the order $\left(\operatorname{Con}_{\mathrm{J}} L ; \leq\right)$ as given in the figure, the methods of G. Czédli [3] and, mainly, [4] are more effective. The prime intervals of $L$ are labeled by the principal congruences they generate. If we represent $\boldsymbol{\alpha}$ and $\boldsymbol{\beta}$ by the thick edges as $\boldsymbol{\alpha}=\operatorname{con}\left(\mathfrak{p}^{\prime}\right)$ and $\boldsymbol{\beta}=\operatorname{con}\left(\mathfrak{q}^{\prime}\right)$, then the General Swing Lemma applied for $\mathfrak{p}^{\prime}$ and $\mathfrak{q}^{\prime}$ yields that $n=0, \mathfrak{p}_{0}=\mathfrak{p}^{\prime}, \mathfrak{q}_{0}=\mathfrak{q}^{\prime}$, and the SPS projectivity described in (1) consist of a single swing of $\mathfrak{p}^{\prime}$ to $\mathfrak{q}^{\prime}$. Hence, we cannot obtain a required covering in $\operatorname{Con}_{\mathrm{J}} L$ in this way. Appropriate $\mathfrak{p}$ and $\mathfrak{q}$ for Lemma 17 are given in the figure, and the proof runs as follows.

Proof of Lemma 17. With $\boldsymbol{\alpha}, \boldsymbol{\beta} \in \mathrm{Con}_{\mathrm{J}} L$ as in the lemma, pick $\boldsymbol{\alpha}^{\prime}$ and $\boldsymbol{\beta}^{\prime}$ in $\mathrm{Con}_{\mathrm{J}} L$ such that $\boldsymbol{\alpha}^{\prime}$ covers $\boldsymbol{\beta}^{\prime}$ in $(\operatorname{Con} L ; \leq), \boldsymbol{\beta} \leq \boldsymbol{\beta}^{\prime}$, and $\boldsymbol{\alpha}^{\prime} \leq \boldsymbol{\alpha}$. Since a join-irreducible congruence of a finite lattice is always generated by a prime interval, we can pick prime intervals $\mathfrak{p}^{\prime}$ and $\mathfrak{q}^{\prime}$ in $L$ such that $\boldsymbol{\alpha}^{\prime}=\operatorname{con}\left(\mathfrak{p}^{\prime}\right)$ and $\boldsymbol{\beta}^{\prime}=\operatorname{con}\left(\mathfrak{q}^{\prime}\right)$. Since $\operatorname{con}\left(\mathfrak{q}^{\prime}\right)=\boldsymbol{\beta}^{\prime}<\boldsymbol{\alpha}^{\prime}=\operatorname{con}\left(\mathfrak{p}^{\prime}\right)$, the prime interval $\mathfrak{q}^{\prime}$ is collapsed by $\operatorname{con}\left(\mathfrak{p}^{\prime}\right)$. Hence, the General Swing Lemma yields a sequence (1) from $\mathfrak{p}^{\prime}$ to $\mathfrak{q}^{\prime}$ such that, for each $j \in\{0, \ldots, n-1\}, \mathfrak{r}_{j}$ is perspective to or swings to or switches to $\mathfrak{r}_{j+1}$. Of course, this sequence satisfies that

$$
\operatorname{con}\left(\mathfrak{p}^{\prime}\right)=\operatorname{con}\left(\mathfrak{r}_{0}\right) \geq \operatorname{con}\left(\mathfrak{r}_{1}\right) \geq \cdots \geq \operatorname{con}\left(\mathfrak{r}_{n-1}\right) \geq \operatorname{con}\left(\mathfrak{r}_{n}\right)=\operatorname{con}\left(\mathfrak{q}^{\prime}\right) .
$$

If for an integer $j$ with $0 \leq j<n$, the prime interval $\mathfrak{r}_{j}$ is perspective to or switches to the prime interval $\mathfrak{r}_{j+1}$, then $\operatorname{con}\left(\mathfrak{r}_{j}\right)=\operatorname{con}\left(\mathfrak{r}_{j+1}\right)$. Therefore, since 
$\operatorname{con}\left(\mathfrak{p}^{\prime}\right)$ covers $\operatorname{con}\left(\mathfrak{q}^{\prime}\right)$ in $\left(\operatorname{Con}_{\mathrm{J}} L ; \leq\right)$ and all the $\operatorname{con}\left(\mathfrak{r}_{j}\right)$ belong to $\operatorname{Con}_{\mathrm{J}} L$, there is a unique $i \in\{1, \ldots, n-1\}$ such that $\operatorname{con}\left(\mathfrak{p}^{\prime}\right)=\operatorname{con}\left(\mathfrak{r}_{i}\right), \mathfrak{r}_{i}$ swings to $\mathfrak{r}_{i+1}$, and $\operatorname{con}\left(\mathfrak{r}_{i+1}\right)=\operatorname{con}\left(\mathfrak{q}^{\prime}\right)$. Therefore, we can let $\mathfrak{p}:=\mathfrak{r}_{i}$ and $\mathfrak{q}:=\mathfrak{r}_{i+1}$.

We conclude this paper with one more variant of the General Swing Lemma. This variant makes no direct reference to planarity.

Let $L$ be a finite lattice and let $m$ be a tab of $L$. Then $K=L-\{m\}$ is a sublattice of $L$. We obtain $K$ by stripping the tab $m$ from $L$. A finite lattice $L$ is stripped, if it has no tabs. It is clear that by consecutive stripping of tabs, we obtain from $L$ a stripped (sub) lattice $L^{\text {s }}$. If we obtain the stripped sublattices $K_{1}$ and $K_{2}$ from the finite lattice $L$, then $K_{1}$ and $K_{2}$ are isomorphic; this follows from the proof of Lemma 4.1 in G. Czédli and E. T. Schmidt [8]. For instance, starting from $\mathrm{M}_{3}$, we obtain three different stripped sublattices, all of them isomorphic to $\mathrm{C}_{2}^{2}$.

Lemma 18 (Reduction Lemma). Let $L$ be a finite semimodular lattice and let $K$ be a stripped sublattice of $L$. If $K$ satisfies the Swing Lemma, then $L$ satisfies the General Swing Lemma.

A proof of the Reduction Lemma is implicit in Section 6. Observing that in the Reduction Lemma, if $L$ is planar and semimodular, then $K$ is slim, planar, and semimodular, we conclude that the Reduction Lemma implies the General Swing Lemma.

Finally, we know that gluing preserves semimodularity; see, for example, E. Fried, G. Grätzer, and E. T. Schmidt [9, Theorem 27] for a stronger statement. Hence, if we glue a non-planar distributive lattice and a planar but non-slim semimodular lattice, then we obtain a nontrivial example in the scope of the Reduction Lemma but not of the General Swing Lemma.

\section{REFERENCES}

[1] Czédli, G.: Representing homomorphisms of distributive lattices as restrictions of congruences of rectangular lattices. Algebra Universalis 67, 313-345 (2012)

[2] Czédli, G.: Diagrams and rectangular extensions of planar semimodular lattices. Algebra Universalis, to appear.

[3] G. Czédli, Patch extensions and trajectory colorings of slim rectangular lattices, Algebra Universalis, 72 (2014), 125-154.

[4] Czédli, G.: A note on congruence lattices of slim semimodular lattices. Algebra Universalis, 72, 225-230 (2014)

[5] G. Czédli and G. Grätzer, Planar Semimodular Lattices: Structure and Diagrams. Chapter 3 in [33].

[6] G. Czédli and G. Makay: Swing lattice game and a short proof of the swing lemma for planar semimodular lattices. Acta Sci. Math. (Szeged)

[7] G. Czédli and E. T. Schmidt, Slim semimodular lattices. I. A visual approach, Order 29 (2012), 481-497.

[8] G. Czédli and E. T. Schmidt, Slim semimodular lattices. II. A description by patchwork systems, Order 30 (2013), 689-721.

[9] E. Fried, G. Grätzer, and E. T. Schmidt, Multipasting of lattices Algebra Universalis 30 (1993), 241-260.

[10] Grätzer, G.: Equational classes of lattices. Duke Math. J. 33 (1966), 613-622. 
[11] Grätzer, G.: Lattice Theory: Foundation. Birkhäuser Verlag, Basel, 2011. xxix+613 pp. ISBN: 978-3-0348-0017-4.

[12] Grätzer, G.: Congruences and prime-perspectivities in finite lattices. Algebra Universalis 74 (2015), 351-359. arXiv: 1312.2537

[13] Grätzer, G.: On a result of Gábor Czédli concerning congruence lattices of planar semimodular lattices. Acta Sci. Math. (Szeged). arXiv 1403.3875

[14] Grätzer, G.: Congruences and trajectories in planar semimodular lattices. Algebra Universalis.

[15] Grätzer, G.: Planar Semimodular Lattices: Congruences. Chapter 4 in [33].

[16] Grätzer, G.: The Congruences of a Finite Lattice, A Proof-by-Picture Approach, second edition. Birkhäuser, 2016. xxxii+347.

[17] Grätzer, G.: Congruences of fork extensions of lattices. Algebra Universalis, published online Aug. 17, 2016.

[18] Grätzer, G.: Homomorphisms and principal congruences of bounded lattices. I. Isotone maps of principal congruences Acta Sci. Math. (Szeged).

[19] Grätzer, G.: Homomorphisms and principal congruences of bounded lattices. II. Sketching the proof for sublattices. Acta Sci. Math. (Szeged).

[20] Grätzer, G.: Homomorphisms and principal congruences of bounded lattices. III. The Independence Theorem. Algebra Universalis.

[21] G. Grätzer, A technical lemma for congruences of finite lattices. Algebra Universalis $\mathbf{7 2}$ (2014), 53-55.

[22] Grätzer, G.: Congruences in slim, planar, semimodular lattices: The Swing Lemma, Acta Sci. Math. (Szeged) 81 (2015), 381-397

[23] G. Grätzer and E. Knapp, Notes on planar semimodular lattices. I. Construction. Acta Sci. Math. (Szeged) $\mathbf{7 3}$ (2007), 445-462.

[24] G. Grätzer and E. Knapp, A note on planar semimodular lattices. Algebra Universalis 58 (2008), 497-499.

[25] Notes on planar semimodular lattices. II. Congruences. Acta Sci. Math. (Szeged) $\mathbf{7 4}$ (2008), 37-47.

[26] Notes on planar semimodular lattices. III. Rectangular lattices. Acta Sci. Math. (Szeged) $\mathbf{7 5}$ (2009), 29-48.

[27] , Notes on planar semimodular lattices. IV. The size of a minimal congruence lattice representation with rectangular lattices. Acta Sci. Math. (Szeged) 76 (2010), $3-26$.

[28] G. Grätzer, H. Lakser, and E. T. Schmidt, Congruence lattices of finite semimodular lattices. Canad. Math. Bull. 41 (1998), 290-297.

[29] G. Grätzer and E. T. Schmidt, Ideals and congruence relations in lattices. Acta Math. Acad. Sci. Hungar. 9 (1958), 137-175.

[30] G. Grätzer and E. T. Schmidt, Congruence lattices of finite semimodular lattices, Abstracts Amer. Math. Soc. 97T-06-56.

[31] G. Grätzer and E. T. Schmidt, A short proof of the congruence representation theorem for semimodular lattices. Algebra Universalis 71 (2014), 65-68.

[32] G. Grätzer and E. T. Schmidt, An extension theorem for planar semimodular lattices. Periodica Mathematica Hungarica (2014) 69 (2014), 32-40.

[33] G. Grätzer and F. Wehrung eds., Lattice Theory: Special Topics and Applications. Volume 1. Birkhäuser Verlag, Basel, 2014

[34] G. Grätzer and F. Wehrung eds., Lattice Theory: Special Topics and Applications. Volume 2. Birkhäuser Verlag, Basel, 2016

\section{GÁBOR CzÉDLI}

University of Szeged, Bolyai Institute, Szeged, Aradi vértanúk tere 1, Hungary 6720

e-mail, G. Czédli: czedli@math.u-szeged.hu

URL: http://www.math.u-szeged.hu/ czedli/

George Grätzer AND Harry LaKser 
Department of Mathematics, University of Manitoba, Winnipeg, MB R3T 2N2, Canada e-mail, G. Grätzer: gratzer@me.com

e-mail, H. Lakser: hlakser@gmail.com

$U R L$, G. Grätzer: http://server.math.umanitoba.ca/homepages/gratzer/ 